International Journal of Bifurcation and Chaos, Vol. 8, No. 12 (1998) 2425-2431

(c) World Scientific Publishing Company

\title{
HIGHER-ORDER SPECTRA OF NONLINEAR POLYNOMIAL MODELS FOR CHUA'S CIRCUIT
}

\author{
STEVE ELGAR* and BARRY VANHOFF \\ School of Electrical Engineering and Computer Science, \\ Washington State University, Pullman, WA 99164-2752, USA \\ LUIS A. AGUIRRE ${ }^{\dagger}$ and UBIRATAN S. FREITAS \\ Centro de Pesquisa e Desenvolvimento em Engenharia Elétrica \\ ${ }^{\dagger}$ Departamento de Engenharia Eletrônica, Universidade Federal de Minas Gerais, \\ Av. Antônio Carlos 6627, 31270-901 Belo Horizonte, M.G., Brazil \\ VINOD CHANDRAN \\ School of Electrical and Electronic Systems Engineering, \\ Queensland University of Technology, Brisbane, Australia
}

Received April 2, 1998; Revised May 14, 1998

\begin{abstract}
Polynomial models are shown to simulate accurately the quadratic and cubic nonlinear interactions (e.g. higher-order spectra) of time series of voltages measured in Chua's circuit. For circuit parameters resulting in a spiral attractor, bispectra and trispectra of the polynomial model are similar to those from the measured time series, suggesting that the individual interactions between triads and quartets of Fourier components that govern the process dynamics are modeled accurately. For parameters that produce the double-scroll attractor, both measured and modeled time series have small bispectra, but nonzero trispectra, consistent with higher-than-second order nonlinearities dominating the chaos.
\end{abstract}

\section{Introduction}

Polynomial models for nonlinear time series reproduce aspects important to the dynamics of the original process from which the model parameters are estimated [Aguirre \& Billings, 1995; Çinar, 1995; Letellier et al., 1997]. Although comparing model time series with original data for systems sensitive to initial conditions is difficult, statistical and bulk properties of polynomial models (such as phase spaces, Poincaré sections, bifurcation diagrams, fixed points, and Lyapunov exponents) can be compared with those of the original data. Here, it is shown that polynomial models estimated from time series sampled from realizations of Chua's circuit [Chua et al., 1993] have higher-order spectra
[Hasselmann et al., 1963; Elgar \& Chandran, 1993] that are similar to those from the measured time series.

The polynomial (NARMAX) model $y(k)$, where $k$ is discrete time (lag) is given by [Leontaritis \& Billings, 1985]

$$
\begin{aligned}
y(k)= & f^{l}\left[y(k-1), \ldots, y\left(k-n_{y}\right),\right. \\
& u(k-d), \ldots, u\left(k-n_{u}\right), \\
& \left.e(k), \ldots, e\left(k-n_{e}\right)\right]
\end{aligned}
$$

where $f^{l}$ is a polynomial of degree $l$, the input $u(k)$ and output $y(k)$ are discretely sampled time series of length $N$, the maximum lags considered for the output, input, and noise terms are $n_{y}, n_{u}$, and $n_{e}$,

*E-mail: elgar@eecs.wsu.edu 
respectively, and $d$ is the delay measured in sampling intervals. Uncertainties, noise, and unmodeled dynamics are accounted for by $e(k)$. Given a time series, polynomial coefficients can be estimated by minimizing errors between model and data [Billings et al., 1989].

Chua's circuit is described by a set of three ordinary differential equations [Chua et al., 1986, 1993; Madan, 1993]

$$
\begin{aligned}
C_{1} \frac{d v_{C_{1}}}{d t} & =\frac{1}{R}\left(v_{C_{2}}-v_{C_{1}}\right)-g\left(v_{C_{1}}\right) \\
C_{2} \frac{d v_{C_{2}}}{d t} & =\frac{1}{R}\left(v_{C_{1}}-v_{C_{2}}\right)+i_{L} \\
L \frac{d i_{L}}{d t} & =-v_{C_{2}}
\end{aligned}
$$

where $v_{C_{1}}$ and $v_{C_{2}}$ are the voltages across capacitors $C_{1}$ and $C_{2}$, respectively, and $i_{L}$ is the current flowing through the inductor. The resistance $(v / i)$ is given by $R$ and $g(\cdot)$ is a piecewise-linear function relating the current in the nonlinear resistor $N_{R}\left(g\left(v_{N_{R}}\right)\right)$ to the voltage $\left(v_{N_{R}}\right)$

$$
\begin{aligned}
g\left(v_{N_{R}}\right)= & m_{0} v_{N_{R}}+0.5\left(m_{1}-m_{0}\right) \\
& \times\left(\left|v_{N_{R}}+B_{p}\right|-\left|v_{N_{R}}-B_{p}\right|\right) .
\end{aligned}
$$

The slope of the current versus voltage curve changes from $m_{0}$ to $m_{1}$ when the voltage changes in absolute value from greater than $B_{p}$ to less than $B_{p}$. Here, the system is moved into different chaotic regimes by changing the resistance $R$, while the other components remain fixed.

The nonlinear interactions between triads and quartets of Fourier components of time series produced by numerical implementations of Chua's circuit (2) are shown by higher-order spectra [Elgar \& Chandran, 1993]. The auto bispectrum is defined formally as the Fourier transform of the third-order correlation function of the time series [Hasselmann et al., 1963]. The discrete bispectrum, appropriate for sampled data, is [Haubrich, 1965; Kim \& Powers, 1979]

$$
B\left(f_{1}, f_{2}\right)=E\left[A_{f_{1}} A_{f_{2}} A_{f_{1}+f_{2}}^{*}\right]
$$

where $A_{f_{i}}$ is the complex Fourier component of the time series at frequency $f_{i}$, asterisk is complex conjugation, and $E[\cdot]$ is the expected-value, or average, operator.

If the three Fourier components in the triple product on the right-hand side of (4) are independent of each other (e.g. if they have random phase relationships such as a time series with Gaussian statistics), the bispectrum is zero. It is convenient to recast the bispectrum into its normalized (by the power at each of the three frequencies in the triad) magnitude, called the squared bicoherence, $b^{2}\left(f_{1}, f_{2}\right)$, which represents the fraction of the power of the triad of Fourier components $\left(f_{1}, f_{2}, f_{1}+f_{2}\right)$ that is owing to quadratic coupling [Kim \& Powers, 1979].

Similar to the bispectrum, the auto trispectrum is defined formally as the Fourier transform of the fourth-order correlation, and the discrete trispectrum is

$$
T\left(f_{1}, f_{2}, f_{3}\right)=E\left[A_{f_{1}} A_{f_{2}} A_{f_{3}} A_{f_{1}+f_{2}+f_{3}}^{*}\right] .
$$

The normalized magnitude of the trispectrum is called the squared tricoherence, $t^{2}\left(f_{1}, f_{2}, f_{3}\right)$, and is a measure of the fraction of the power of the quartet of Fourier components $\left(f_{1}, f_{2}, f_{3}, f_{1}+f_{2}+f_{3}\right)$ that is owing to cubic nonlinear interactions. Further details, additional references, and tutorial examples of bispectra and trispectra can be found in [Elgar \& Chandran, 1993].

\section{Results}

Time series of voltage $v_{C_{1}}$ measured from a realization of Chua's circuit [Chua et al., 1993] for the spiral [Thompson \& Stewart, 1986] and double-scroll [Chua et al., 1986] attractors were used to estimate the coefficients (given in the Appendix) of the nonlinear polynomial models (1). The circuit parameters used during the experiments were $C_{1}=11 \mu \mathrm{F}$, $C_{2}=45 \mu \mathrm{F}, L=20 \mathrm{H}, B_{p}=1.1 \pm 0.2 \mathrm{~V}$, $m_{0}=-0.37 \pm 0.04 \mathrm{mS}$, and $m_{1}=-0.68 \pm 0.04 \mathrm{mS}$. For the spiral attractor (sampled at $12 \mu \mathrm{s}$ ) $R=$ $1.67 \mathrm{k} \Omega$ and for the double scroll (sampled at $15 \mu \mathrm{s}$ ) $R=1.64 \mathrm{k} \Omega$. The high inductance (achieved by implementing an OPAMP-based circuit that mimics the dynamics of an inductor) reduces the oscillation frequency from $O(100 \mathrm{kHz})$ to $O(1 \mathrm{kHz})$, thus facilitating A/D conversion and recording of the data. Polynomial model parameters (Appendix) were estimated from 8192 values of the voltage measured after initial transients had decayed.

Both the measured and modeled spiral attractors have a relatively narrow power spectrum dominated by a primary peak $f_{p}=2.92 \mathrm{~Hz}$ and its harmonics $\left[2 f_{p}=5.85\right.$ and $3 f_{p}=8.78 \mathrm{~Hz}$, Fig. 1(a)]. The model bicoherence [Fig. 1(c)] indicates quadratic coupling between the primary and 

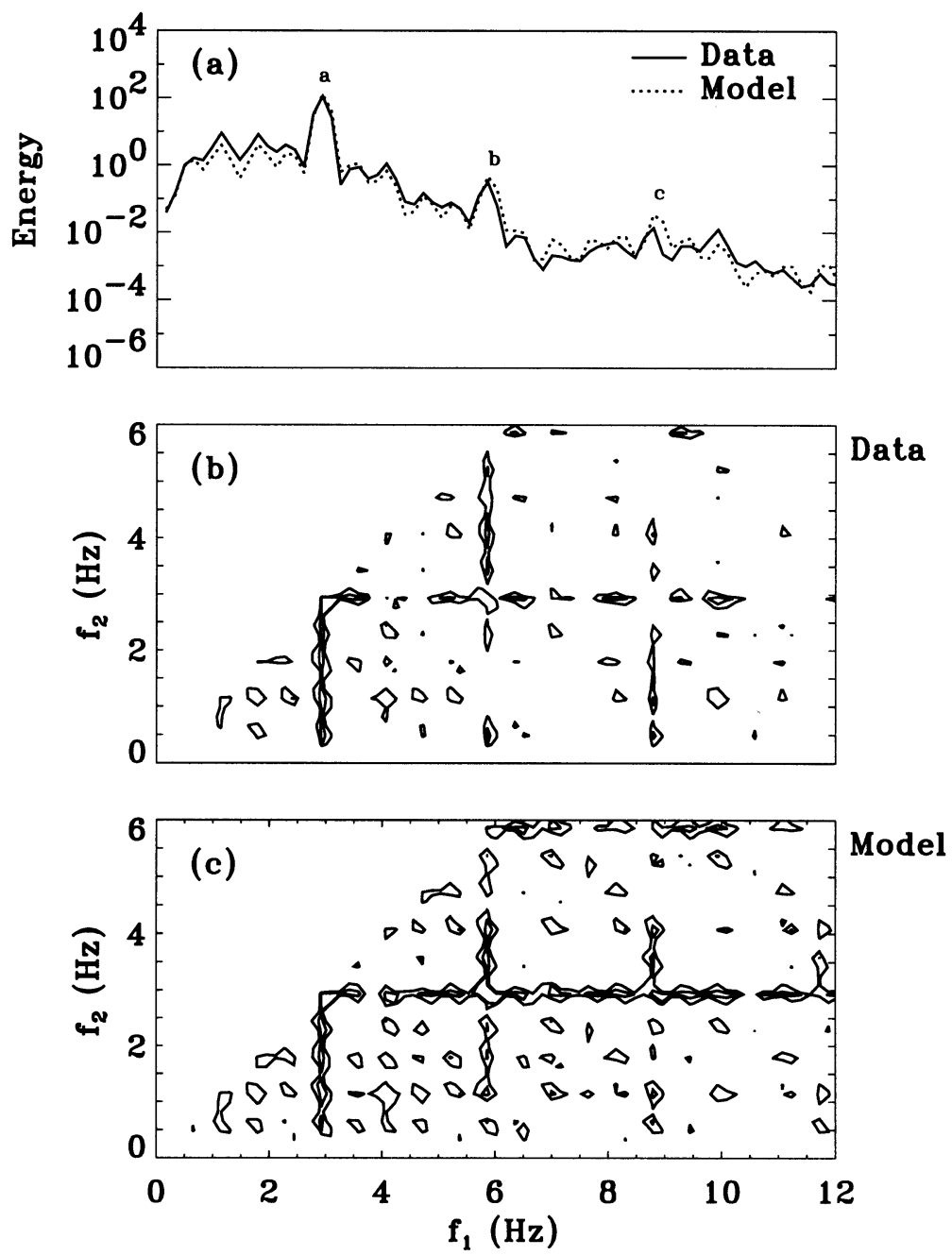

Fig. 1. (a) Power spectra of measured (solid curve) and modeled (dashed curve) voltage $v_{c_{1}}$ from Chua's circuit when the system exhibits a spiral attractor. Contours of bicoherence of (b) measured and (c) modeled voltage $v_{c_{1}}$. The units of power are arbitrary and the frequencies of the labeled power spectral peaks (panel a) are (a) 2.92, (b) 5.85, and (c) 8.78 Hz. The minimum contour plotted is $b=0.85$, with additional contours every 0.1 . There are 32 degrees of freedom.

its harmonics [e.g. the horizontal band of contours for $f_{2} \approx 3 \mathrm{~Hz}$, Fig. 1(c)], similar to that observed in the measurements [Fig. 1(b)]. Quadratic interactions involving Fourier components with frequencies less than $f_{p}$ are significant in time series from both the data $\left[1<f_{2}<3, f_{1} \approx 3 \mathrm{~Hz}\right.$, Fig. $\left.1(\mathrm{~b})\right]$ and the model [Fig. 1(c)]. Tricoherence spectra for the measured time series [Figs. 2(a)-2(c)] are similar to those from the polynomial model [Figs. 2(d)-2(f)]. Cubic interactions between quartets of Fourier components are significant for the spiral attractor, suggesting higher-order coupling between motions with frequencies $f_{p}$ and its harmonics. The coupling between the primary and its harmonics, as indicated by the bicoherence [Figs. 1(b) and 1(c)] and tricoherence (Fig. 2) spectra, suggest that both quadratic and cubic interactions (as occur in the spiral attractor) are reproduced well by the polynomial model estimated from 8192 values of the voltage measured in Chua's circuit.

The higher-order spectral coherences from the model time series are slightly higher than those from the data, possibly because an infinite-order polynomial is required to reproduce exactly the piecewiselinear current in the nonlinear resistor (3), and thus the second- and third-order polynomial model used here may have stronger quadratic and cubic coupling than observed in the actual circuit. Noise in the measured circuit may also reduce higher-order spectral values relative to those from the model time series.

The power spectrum for the measured doublescroll attractor (Fig. 3) is broader than the spiral attractor [Fig. 1(a)], with a relatively small 
Data
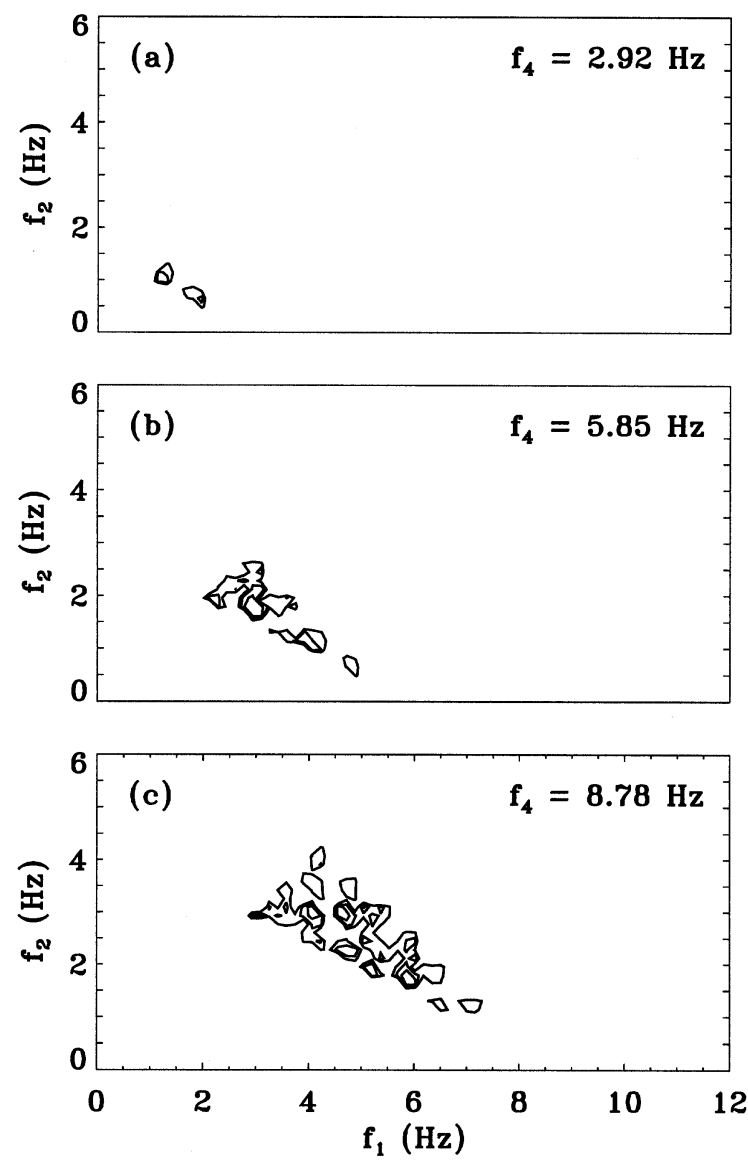

Model
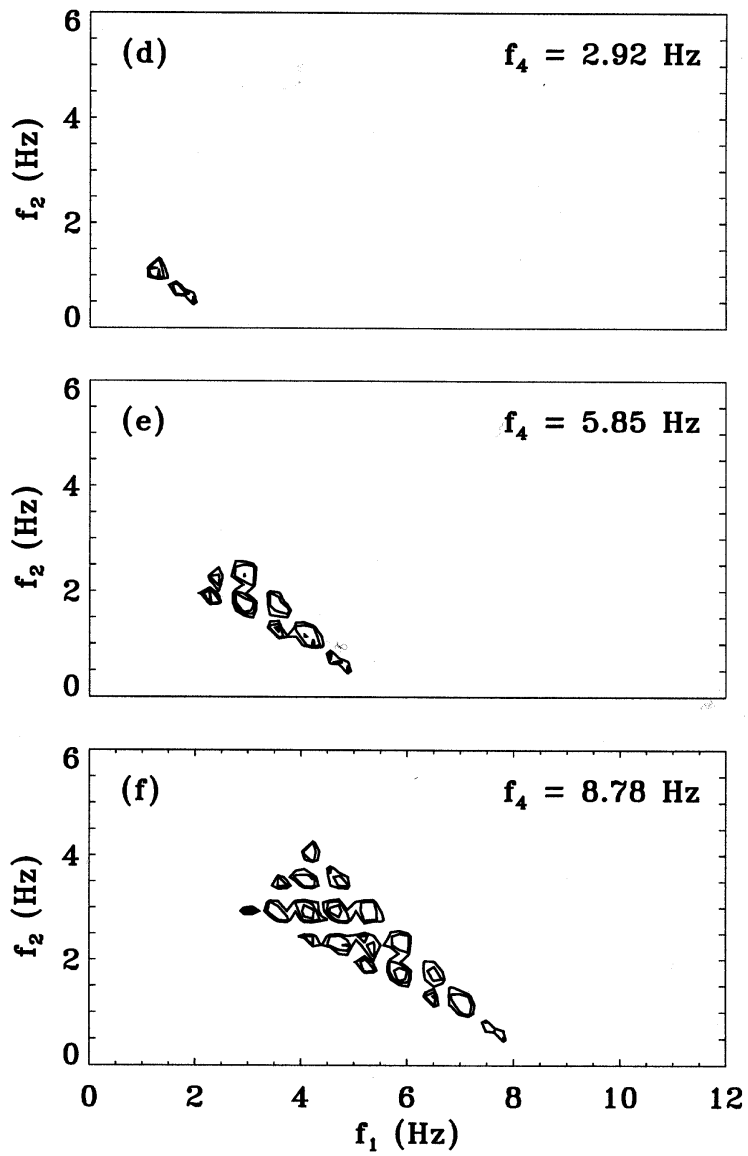

Fig. 2. Tricoherence spectra of the measured voltage $v_{c_{1}}$ from Chua's circuit (a)-(c) and from a polynomial model of the circuit (d)-(f) when the system exhibits a spiral attractor. The constant sum frequencies are (a) and (d) $f_{4}=2.92,(\mathrm{~b})$ and (e) $f_{4}=5.85$, and (c) and (f) $f_{4}=8.78 \mathrm{~Hz}$. (See [Elgar \& Chandran, 1993] for a description of the plot format.) The minimum contour plotted is $t=0.61$, with additional contours every 0.11 . There are 32 degrees of freedom.

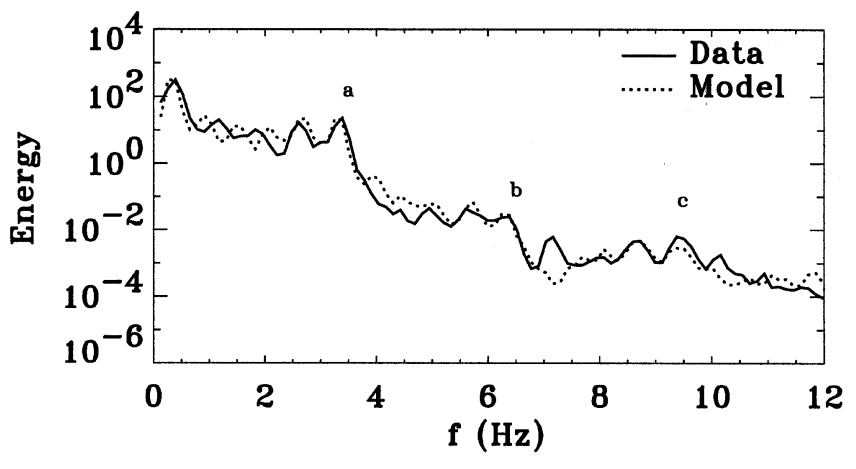

Fig. 3. Power spectra of measured (solid curve) and modeled (dashed curve) voltage $v_{c_{1}}$ from Chua's circuit when the system exhibits the double-scroll attractor. The units of power are arbitrary and the frequencies of the labeled power spectral peaks are (a) 3.38, (b) 6.38, and (c) $9.37 \mathrm{~Hz}$. There are 32 degrees of freedom. primary peak $\left(f_{p}=3.38 \mathrm{~Hz}\right)$ and more energetic low-frequency motions. The polynomial model shows similar power spectral features (Fig. 3). There are no statistically significant bicoherences (not shown) in the measured Chua circuit or in the polynomial model, indicating that the nonlinearities for the double-scroll are not quadratic. This result is consistent with cluster analysis of similar attractors [Aguirre et al., 1997; Aguirre, 1997]. As shown by the tricoherence spectrum (Fig. 4), many of the Fourier components of the measured double-scroll system are cubically coupled to each other, and furthermore, interactions involving low frequency components are important to the dynamics (i.e. there are many cubically coupled triads involving low frequencies, e.g. $f_{2}<2 \mathrm{~Hz}$ in 
Data
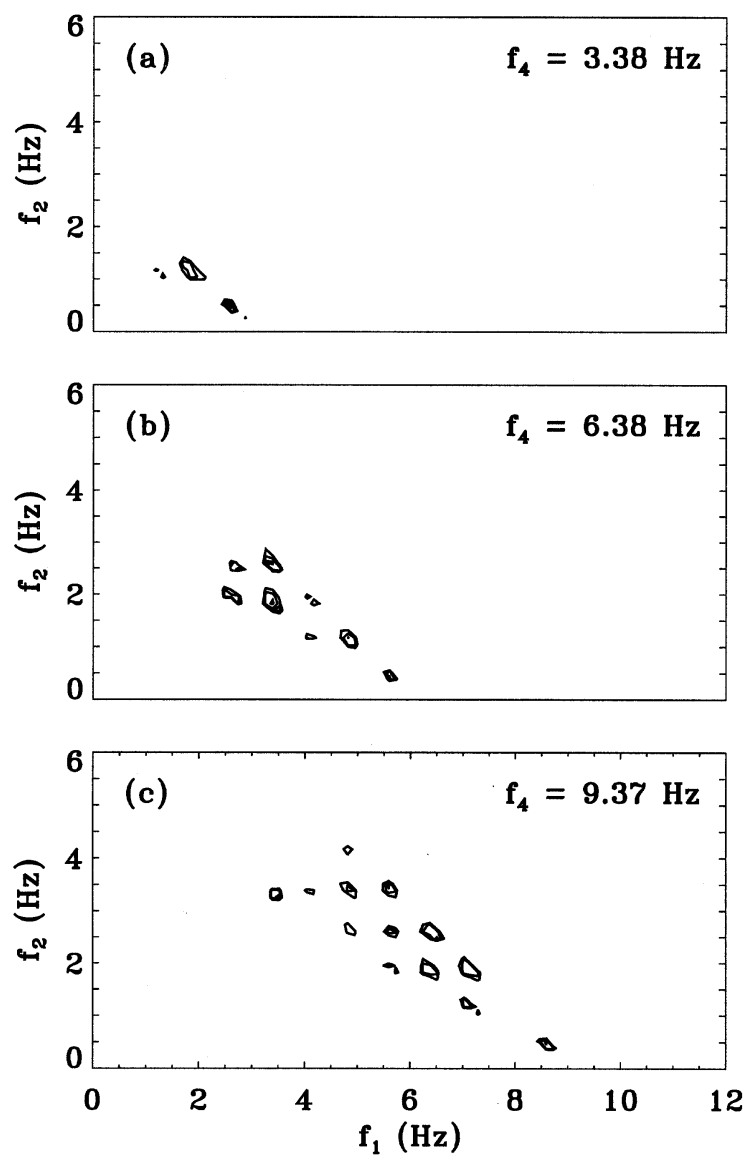

Model
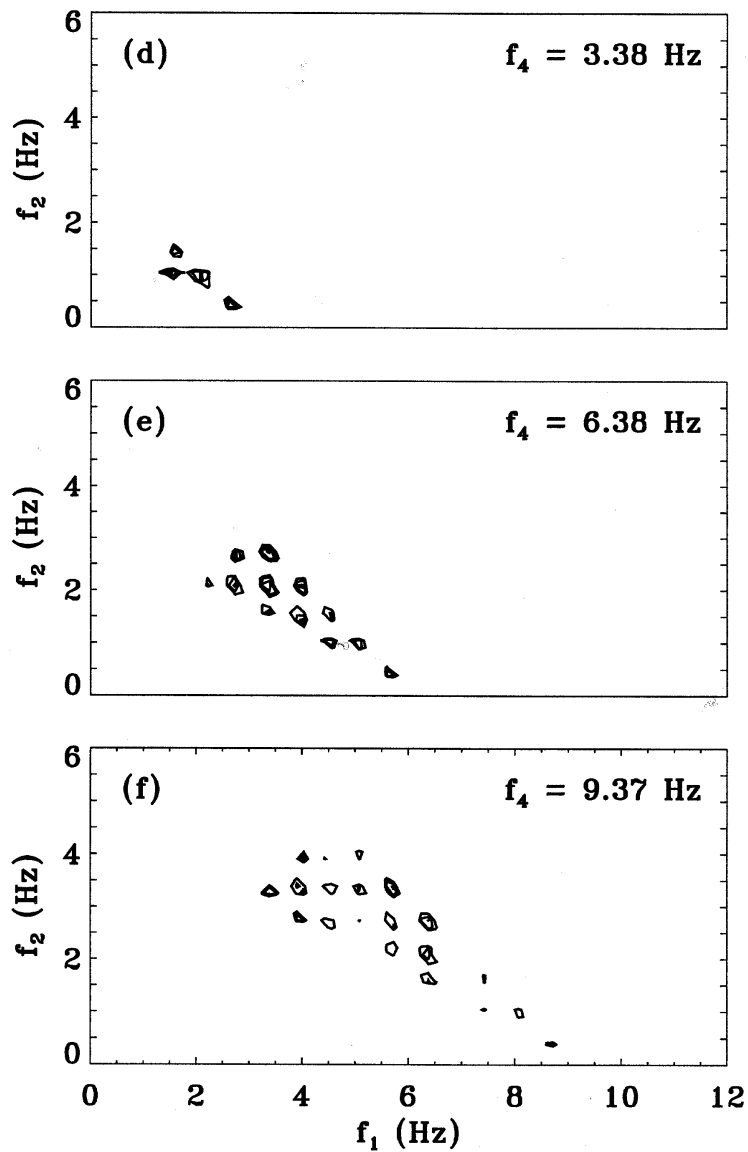

Fig. 4. Tricoherence spectra of the measured voltage $v_{c_{1}}$ from Chua's circuit (a)-(c) and from a polynomial model of the circuit (d)-(f) when the system exhibits a double-scroll attractor. The constant sum frequencies are (a) and (d) $f_{4}=3.38$, (b) and (e) $f_{4}=6.38$, and (c) and (f) $f_{4}=9.37 \mathrm{~Hz}$. The minimum contour plotted is $t=0.61$, with additional contours every 0.11 . There are 32 degrees of freedom.

Figs. 4(a)-4(c). The tricoherence spectrum of the polynomial model shows similar features [Figs. 4(d)-4(f)], suggesting the measured cubic coupling between the primary and its harmonics is reproduced well by the polynomial model.

\section{Conclusions}

Higher-order spectral analysis suggests that polynomial models of Chua's circuit have quadratic and cubic nonlinear interactions similar to those observed in the underlying time series from which the model parameters are estimated. The results presented here agree with the cluster analyses of chaotic attractors produced by the Chua circuit, and suggest that higher-order spectra can aid in the structure selection of polynomial models.

\section{Acknowledgments}

The research was supported by the Office of Naval Research (USA) and CNPq (Brazil). The authors are grateful to Leonardo Torres for designing the OPAMP-based circuit.

\section{References}

Aguirre, L. A. [1997] "On the structure of nonlinear polynomial models: Higher-order correlation functions, spectra, and term clusters," IEEE Trans. Circuits Syst. I: Fundamental Theor. Appl. 44(5), 450-453.

Aguirre, L. A. \& Billings, S. A. [1995] "Retrieving dynamical invariants from chaotic data using NARMAX models," Int. J. Bifurcation and Chaos 5(2), 449-474. Aguirre, L. A., Rodrigues, G. G. \& Mendes, E. M. A. M. [1997] "Nonlinear identification and cluster analysis of chaotic attractors from a real implementation of 
Chua's circuit," Int. J. Bifurcation and Chaos 7(6), 1411-1423.

Billings, S. A., Chen, S. \& Korenberg, M. [1989] "Identification of MIMO nonlinear systems using a forward-regression orthogonal estimator," Int. J. Control 49(6), 2157-2189.

Chua, L. O., Komuro, M. \& Matsumoto, T. [1986] "The double-scroll family," IEEE Trans. Circuits Syst. I: Fundamental Theor. Appl. 33, 1073-1118.

Chua, L. O., Wu, C. W., Huang, A. \& Zhong, G. Q. [1993] "A universal circuit for studying and generating chaos, Part I: Routes to chaos," Special Issue on Chaos in Electronic Circuits, Part A, IEEE Trans. Circuits Syst. I: Fundamental Theor. Appl. 40(10), 732-744.

Çinar, A. [1995] "Nonlinear time series models for multivariable dynamic processes," Chemometrics Intell. Lab. Syst. 30, 147-158.

Elgar, S. \& Chandran, V. [1993] "Higher-order spectral analysis to detect nonlinear interactions in measured time series and an application to Chua's circuit," Int. J. Bifurcation and Chaos 3(1), 19-34.

Hasselmann, K., Munk, W. \& MacDonald, G. [1963] "Bispectra of ocean waves," in Time Series Analysis, ed. Rosenblatt, M. (Wiley, NY), pp. 125-139.

Haubrich, R. A. [1965] "Earth noise, 5 to 500 millicycles per second," J. Geophys. Res. 70(6), 1415-1427.

Kim, Y. C. \& Powers, E. J. [1979] "Digital bispectral analysis and its applications to nonlinear wave interactions," IEEE Trans. Plasma Sci. 7(2), 120-131.
Leontaritis, I. J. \& Billings, S. A. [1985] "Input-output parametric models for nonlinear systems Part II: Stochastic nonlinear systems," Int. J. Control 41(2), 329-344.

Letellier, C., Le Sceller, L., Gouesbet, G., Lusseyran, F., Kemoun, A. \& Izrar, B. [1997] "Recovering deterministic behavior from experimental time series in mixing reactor," AIChE 43(9), 2194-2202.

Madan, R. N. [1993] Chua's Circuit: A Paradigm for Chaos (World Scientific, Singapore).

Thompson, J. B. \& Stewart, H. B. [1986] Nonlinear Dynamics and Chaos (Wiley, NY), pp. 235-238.

\section{Appendix}

Models were selected based on (i) stability, (ii) geometry of the reconstructed attractor (visual analysis), (iii) largest Lyapunov exponent, and (iv) correlation dimension. Unstable models and stable models with orbits in phase space differing visually from orbits produced by the measured time series were rejected. Similarly, models with largest Lyapunov exponent and correlation dimension differing significantly from those of the measured time series were rejected. Polynomial models not rejected by these criteria that were used to reconstruct the spiral and double-scroll attractors are given, respectively, by

$$
\begin{aligned}
y(k)= & 1.0492699 \times 10^{-3}+3.8820073 y(k-1)-5.3586346 y(k-2)+3.2272437 y(k-3) \\
& -6.7316594 \times 10^{-1} y(k-4)-1.0908277 y^{2}(k-1)+1.8120221 y(k-1) y(k-2) \\
& +1.2853315 \times 10^{-1} y^{2}(k-2)-5.3797216 \times 10^{-2} y^{3}(k-6) \\
& -2.4424720 y^{2}(k-3)+3.4350846 y(k-3) y(k-4)-1.3226760 y^{2}(k-4) \\
& -5.7482072 \times 10^{-1} y(k-1) y(k-4)-2.7943574 \times 10^{-2} y^{2}(k-4) y(k-5) \\
& +7.0459715 \times 10^{-3} y^{3}(k-1)+8.3729350 \times 10^{-2} y(k-5) y^{2}(k-6) \\
& +\sum_{i=1}^{19} \hat{\theta}_{i} \xi(k-i)+\xi(k),
\end{aligned}
$$

and

$$
\begin{aligned}
y(k)= & 3.4100533 y(k-1)-4.1012259 y(k-2)+2.1424854 y(k-3) \\
& -5.5119040 \times 10^{-1} y(k-4)-1.5660850 y^{3}(k-1)+1.2719477 \times 10^{-1} y(k-5) \\
& +3.7094852 y^{2}(k-1) y(k-2)-7.1252117 \times 10^{-1} y(k-1) y(k-2) y(k-3) \\
& +7.2200953 \times 10^{-1} y^{2}(k-2) y(k-4)-4.9131975 \times 10^{-2} y^{3}(k-5) \\
& +2.3934928 \times 10^{-1} y(k-3) y^{2}(k-5)-1.1878823 \times 10^{-1} y(k-2) y(k-3) y(k-5) \\
& -1.7485641 y(k-1) y^{2}(k-2)-3.1117395 \times 10^{-1} y^{2}(k-1) y(k-4) \\
& -1.7004093 \times 10^{-1} y^{2}(k-4) y(k-5)+\sum_{i=1}^{19} \hat{\theta}_{i} \xi(k-i)+\xi(k),
\end{aligned}
$$


where the summation represents the moving average part of the model, fitted during parameter estimation to avoid bias (but not used to produce the time series analyzed here), and $\xi(k)$ are the residuals (prediction errors). The terms selected explain the maximum amount of variance given the model constraints. The first 8192 values of each model simulation were discarded to avoid transients, and time series from the next 8192 values were used in Figs. 1-4. The power and higher-order spectra are not affected significantly by changes in the processing details (e.g. using more or fewer values, obtaining statistical stability with frequency merging or ensemble averaging), nor by using different sets of 8192 values (after the transient). The largest Lyapunov exponents for the spiral attractor are $1.31 \times 10^{-2}$ and $1.63 \times 10^{-2}$ for data and model (A.1), respectively. The largest Lyapunov exponents for the double-scroll attractor are $3.30 \times$ $10^{-2}$ and $2.84 \times 10^{-2}$ for data and model (A.2), respectively. 